\title{
El interjuego entre el compromiso organizacional y los valores personales
}

Alicia Omar ${ }^{*}$, Laura Paris ${ }^{* *}$, Juan Diego Vaamonde ${ }^{* \star *}$

\section{Resumen}

El estudio del compromiso organizacional (CO) está capturando la atención de gerentes e investigadores. Además de ser una de las alternativas más eficaces para la consecución de las metas organizacionales, la importancia del CO radica en su capacidad para influir en la eficiencia y el bienestar de los miembros de una organización. El objetivo del presente trabajo fue explorar las relaciones entre el CO y los valores individuales en una muestra de empleados argentinos. Los sujetos completaron una hoja de datos personales, la escala de Individualismo-Colectivismo (vertical y horizontal) y la de Compromiso Organizacional. La muestra total fue clasificada en 8 subgrupos, en función de la dicotomización por la mediana de las cuatro dimensiones axiológicas (IH: individualismo horizontal, IV: individualismo vertical, $\mathrm{CH}$ : colectivismo horizontal y CV: colectivismo vertical). Se seleccionaron los 4 subgrupos más representativos ( $\mathrm{N}=162$ ), vale decir, los que exhibían altos puntajes en una de las cuatro dimensiones axiológicas y bajos en las restantes tres. En tales grupos se calcularon análisis de varianza simples, pruebas de comparación a posteriori y análisis de correlación entre las variables exploradas. Los resultados mostraron que: a) los colectivistas (CH ó CV), desarrollan mayor nivel de compromiso afectivo, mientras que los individualistas (IH ó IV), se vinculan con sus organizaciones a través del compromiso normativo y/o calculativo; b) las mujeres y los empleados con mayor antigüedad exhiben mayores niveles de compromiso afectivo y normativo; c) los más escolarizados, los que ocupan cargos gerenciales y los empleados de grandes empresas se sienten unidos a sus organizaciones a través del compromiso normativo, y d) el compromiso calculativo pareciera operar de modo diferente al afectivo y al normativo, desde el momento que no aparece vinculado con ninguna de las variables sociodemográficas exploradas. Se analizan las limitaciones del estudio y se proponen alternativas superadoras para futuras investigaciones en el área.

Palabras clave: compromiso organizacional, colectivismo, individualismo, horizontal, vertical.

\footnotetext{
* CONICET-UNR, Universidad Nacional de Rosario - Italia 1365, $1^{\circ}$ A (2000) Rosario, Argentina. Email: agomar@arnet.com.ar

** Facultad de Derecho y Ciencias Sociales del Rosario, Universidad Católica. Argentina.

*** Facultad de Psicología, Universidad Nacional de Rosario. Argentina
} 


\section{Abstract}

\section{The interplay between organizational commitment and personal values}

The study of employee's commitment is capturing the attention of managers and researchers. Besides being one of the most effective alternatives for achieving the organizational goals, the importance of organizational commitment (OC) lies in its ability to influence the efficiency and the wellbeing of members of an organization. The article examines the relationships between personal values and components of OC. Participants were selected from twelve Argentinean organizations. Subjects completed a questionnaire of personal data, and two scales to measure individualismcollectivism (vertical and horizontal), and organizational commitment, respectively. All subjects were classified into eight subgroups, after dichotomizing by median each dimensions of values (HI: horizontal individualism; VI: vertical individualism; HC: horizontal collectivism, and HV: vertical collectivism). The four most representative subgroups $(\mathrm{N}=162)$ were selected, that is, who exhibited high scores in one of the four dimensions and lows in the remaining three. In such subgroups were calculated analysis of variance, post-hoc comparison tests, and correlation analysis. The more significant findings can be summarized in the following items: a) collectivists (HC or VC) show a higher level of affective commitment, while individualists relate to their companies through normative or continuance commitment; b) women and senior employees exhibit greater levels of affective and normative commitment; c) managers, employees with higher academic degree, and employees of large companies, feel attached to their organizations through normative commitment; d) continuance commitment seems to work differently than affective and normative commitment because it has no associations with any of the socio-demographic variables studied. Strengths and limitations of the study are discussed, and suggestions are made for future research.

Key words: organizational commitment, collectivism, individualism, horizontal, vertical. 
El grado de compromiso del empleado con la empresa donde trabaja se ha convertido en uno de los fenómenos más estudiados en los últimos años. Una de las razones de tal interés es que los gerentes y directivos han comenzado a darse cuenta que para lograr ventajas competitivas en una economía global es indispensable contar con recursos humanos identificados con los objetivos de la empresa. Además de ser una de las alternativas más eficaces para la consecución de las metas organizacionales, la importancia del compromiso organizacional (CO) radica en su capacidad para influir sobre la eficiencia y el bienestar de los miembros de una organización. El concepto se refiere al grado con que las personas se identifican, están consustanciadas con sus organizaciones, y están dispuestas a continuar trabajando en ellas (Allen \& Meyer, 1996). Se ha observado que el compromiso que un empleado tiene con su organización se vincula estrechamente con su comportamiento en el lugar de trabajo. No obstante, la naturaleza y dirección de tales relaciones son complejas y dependen del contexto y de las variables en estudio (Meyer, Stanley, Herscovitch, \& Topolnytsky, 2002).

Inicialmente, el constructo fue considerado como unidimensional, ya que se ponía el énfasis casi exclusivamente sobre el apego afectivo a la organización (Mathieu \& Zajack, 1990). No obstante, la concepción del CO como un constructo multidimensional se ha ido fortaleciendo paulatinamente (Greenberg \& Baron, 1997). El modelo propuesto por Meyer y Allen (1991) se encuadra en esta perspectiva. Estos autores conciben al compromiso como un estado psicológico que caracteriza la relación del empleado con su organización y que tiene implicancias en su decisión de mantener o no su condición de miembro de esa organización. Sintetizando las evidencias reunidas por estudios anteriores, Meyer, Allen y Smith (1993) sostuvieron que el CO está constituido por tres factores separados, asociados a la vinculación afectiva con la organización, a la percepción de los costos asociados a dejar el empleo y a la obligación de permanecer en ella. Tales factores fueron designados, respectivamente, como: compromiso afectivo, calculativo y normativo. En síntesis, desde la óptica de Meyer y sus colaboradores, el CO es una relación que se define por el deseo, la necesidad y la obligación de permanecer en la organización.

El compromiso afectivo fue definido como los lazos emocionales que unen al individuo con la organización. Allen y Meyer señalaron que en el proceso de formación de tales lazos interviene la valorización que hace el empleado del apoyo y los beneficios recibidos por parte de la empresa. Si sus evaluaciones son positivas, comenzará a percibir los objetivos de la empresa como si fueran sus propios objetivos y deseará seguir perteneciendo a la misma porque la considera parte de su vida. El compromiso calculativo fue considerado como la toma de conciencia por parte del empleado de los altos costos que generaría el hecho de dejar la organización. El proceso de formación de este tipo de compromiso se basa en un análisis costo-beneficio. Antes de tomar una decisión, el empleado evaluará los sacrificios realizados hasta la fecha, los perjuicios que traería para su familia si dejara el empleo y las posibilidades concretas de encontrar un nuevo empleo frente a las alternativas laborales disponibles en el mercado. Si como 
resultado de tales evaluaciones surgiera que los costos de dejar la organización son mayores que los beneficios, entonces optará por permanecer en la empresa. Seguirá perteneciendo por una cuestión de necesidad o por que precisa hacerlo, más que por un deseo genuino. Su vínculo con la empresa será producto de una decisión calculada por lo que su pertenencia a la empresa estará sustentada en un compromiso de tipo calculativo. Finalmente, el compromiso normativo fue definido como el sentimiento de deber o la obligación de permanecer en la organización. En la formación de este tipo de compromiso tiene un peso preponderante el proceso de socialización al que estuvo sometido el sujeto. Vale decir, el respeto por las normas, la valorización de los grupos de pertenencia, la lealtad al empleador y el reconocimiento de las inversiones que ha realizado la empresa en su desarrollo (por ejemplo, gastos en capacitación y entrenamiento, asistencia para la educación de sus hijos, etc.). Esta presión normativa genera en el empleado sentimientos de retribución que se traducen en la obligación (o el deber) de seguir perteneciendo a la empresa como una forma de reconocimiento. Su vínculo con la organización no será el resultado del deseo ni la conveniencia, sino que reflejará un sentimiento de deber u obligación.

Luego de identificar y definir cada una de las dimensiones del CO, Meyer y Allen señalaron que tales dimensiones pueden ser vivenciadas al mismo tiempo en diferentes grados, aunque, por lo general, siempre prevalece un tipo de CO que vincula al empleado con su empresa. Indicaron también que el compromiso afectivo sería el que tendría consecuencias más positivas para la salud mental del empleado y para el desarrollo de la empresa. Desde el momento que los empleados que establecen relaciones afectivas, basadas en experiencias positivas y gratificantes vividas en el seno de su organización, serían los más satisfechos y los más dispuestos a sacrificarse para el logro de las metas organizacionales. Por lo que dejaron entrever que toda organización que se esfuerza por desarrollar el compromiso afectivo de sus miembros se vería gratificada con un plantel de empleados más felices y más consustanciados con la empresa, con las consecuentes ventajas corporativas que esto trae aparejado.

Como resultado del proceso de validación empírica de su modelo tridimensional, Allen y Meyer (1996) desarrollaron un instrumento para explorar el CO, integrado por 18 ítems (6 para cada dimensión). Estudios recientes han encontrado que las facetas afectiva y normativa del CO se correlacionan positivamente, y ambas surgen como preeditores de comportamientos organizacionalmente positivos, incluyendo aumentos en la calidad del trabajo, en la satisfacción laboral, el bienestar subjetivo, la permanencia y la asistencia (Meyer et al., 2002). El compromiso calculativo, por otro lado, no presenta correlaciones con el compromiso afectivo y se vincula negativamente con conductas laborales positivas. La única similitud entre los tres componentes del CO parece ser su vinculación con bajas intenciones de renunciar (o cogniciones de repliegue), aunque los resultados de un meta-análisis conducido por Meyer et al. (2002) sugieren que no existe una fuerte relación entre el compromiso calculativo y las intenciones de renunciar. Por lo que la pregunta que ahora está instalada es si el compromiso calculativo 
puede ser considerado como una verdadera faceta del CO. Sobre todo porque el CO fue definido (Allen \& Meyer, 1996) como un estado psicológico que une al individuo con la organización y, llamativamente, el compromiso calculativo no necesariamente supone un involucramiento psicológico (Virtanen, 2000). Simultáneamente a los estudios orientados a la operacionalización del constructo, la investigación sobre $\mathrm{CO}$ se viene preocupando por identificar los antecedentes y correlatos de cada una de sus facetas. De particular interés para el presente trabajo, son las investigaciones referidas al impacto de los valores personales sobre el compromiso.

\section{Valores individuales y compromiso organizacional}

Desde la perspectiva de Hofstede (1980), tanto en el seno de las naciones como en el de las organizaciones, los miembros comparten programas mentales que son característicos de sus culturas específicas, por lo que existe una considerable homogeneidad con respecto a los valores dentro de una nación o una organización. Perspectiva, que ha llevado a Trice y Beyer (1993) a señalar que aunque las organizaciones se esfuercen por crear su propia cultura, influencias nacionales, ocupacionales e industriales afectan sistemáticamente los valores organizacionales, ya sea porque son importadas a través de sus miembros, ya porque las organizaciones se adaptan a su entorno. Al respecto, Schwartz (1994) sostiene que las organizaciones se acomodan a los valores de sus miembros para que los individuos puedan funcionar efectivamente dentro de ellas y que si bien puede haber diferencias en valores entre organizaciones de un país, las diferencias entre países son mayores que las observadas en el interior de cada país. Por lo que Jabes y Gruere (1986, citados por Parkes, Bochner \& Schneider, 2001), argumentan que los miembros de una organización manejan valores que tienden a ser, en promedio, similares a los de su cultura nacional.

En líneas generales, el estudio de la cultura nacional se realiza principalmente a través de ciertas dimensiones de valores básicos derivados de análisis teóricos y estudios empíricos, siendo las de individualismo-colectivismo, distancia jerárquica al poder, masculinidad y evitación de la inseguridad (Hofstede, 1980), las dimensiones axiológicas que gozan de mayor consenso entre los estudiosos, $y$, básicamente, la de individualismo-colectivismo (I-C), la más analizada en relación con los valores asociados al trabajo (Alavi \& McCormick, 2004). En su extenso programa sobre los valores humanos, Triandis (1995) definió al individualismo y al colectivismo como síndromes culturales, consistentes en compartir actitudes, creencias, normas, papeles y definiciones del yo. Según Triandis, las culturas individualistas se caracterizan por valorar la autonomía del individuo y su independencia emocional de los grupos sociales, mientras que las sociedades colectivistas rescatan la tradición heredada y privilegian los intereses grupales. Paralelamente, efectuó la distinción entre dos diferentes niveles (Triandis, 1995) a través de los cuales se manifiesta el individualismo-colectivismo: el cultural, 
que hace referencia al énfasis que los grupos culturales conceden a la vida individual o grupal, y el individual, que se refiere al conjunto de sentimientos, creencias y actitudes individuales relacionadas a la preocupación o no por el otro. La tendencia predominante en las investigaciones recientes (Clugston, Howell, \& Dorfman, 2000; Ramamoorthy \& Flood, 2002) es, precisamente, estudiar al individualismo y al colectivismo desde la perspectiva individual.

Si bien la dimensión individualismo-colectivismo fue introducida en la literatura como una variable unidimensional (Hofstede, 1980), a partir de la obra de Triandis (1995) se comenzó a considerar al individualismo y al colectivismo como dos factores independientes, lo que hizo abandonar la idea vigente hasta ese momento de que constituían los polos opuestos de un mismo constructo. Esta reconceptualización permitió sostener que cada sujeto puede presentar características de ambas orientaciones en diferentes proporciones, siendo muchas veces el contexto el que va a definir el estilo de comportamiento prevaleciente (Triandis, Chen, \& Chan, 1998). En esta línea argumental, Dutta-Bergman y Wells (2002) han provisto amplia evidencia sobre variaciones dentro de las culturas en términos de individualismo y colectivismo y las consecuentes diferencias exhibidas con respecto a indicadores comportamentales. Triandis observó, además, la existencia de diferentes tipos de individualismo y colectivismo, y con el propósito de caracterizarlos, introdujo los atributos de horizontal y vertical (Triandis, 1995; 2001), propios de la dimensión distancia al poder de Hofstede (1980). La distancia al poder fue definida por Hofstede como el grado en que los miembros menos poderosos de las instituciones y organizaciones de un país 'aceptan que el poder se distribuya desigualmente' (1980, p. 28). Vale decir que mientras que el individualismo enfoca sobre diferencias humanas, la distancia al poder focaliza sobre cuán ampliamente esas diferencias individuales están relacionadas con otras desigualdades (en poder, riqueza o prestigio) y hasta qué punto tales desigualdades son vistas como legítimas o ilegítimas. En esencia, desde la perspectiva de Triandis, tanto individualismo como colectivismo pueden ser horizontal (si enfatizan la igualdad) o vertical (si enfatizan las jerarquías).

El concepto de horizontal sugiere que las personas son similares en la mayoría de los aspectos, especialmente en estatus. Para los individualistas, esto se traduce en un yo independiente, aunque similar a los demás, posiblemente por el alto nivel de libertad y de igualdad que los caracteriza. Por lo que las personas que se orientan al individualismo horizontal ( $\mathrm{IH}$ ) disfrutan de la privacidad, la independencia y se perciben como siendo iguales a otros. Para los colectivistas, en cambio, esto se traduce en un yo interdependiente, aunque similar a los demás, debido a la baja libertad y alta igualdad que los identifica. Por lo que las personas que se orientan al colectivismo horizontal $(\mathrm{CH})$ se perciben similares a los demás miembros de su grupo, enfatizan los objetivos comunes, disfrutan colaborando con sus compañeros y se esfuerzan por mantener la armonía grupal.

El concepto de vertical sugiere que existen diferencias entre las personas especialmente en estatus y jerarquías. Para los individualistas, esto se refleja en 
un yo independiente y diferente de los demás, posiblemente por que aceptan una baja igualdad aunque le dan máxima importancia al sentido de libertad. Las personas que se orientan al individualismo vertical (IV) quieren diferenciarse de las demás adquiriendo estatus social, son competitivas y les cuesta aceptar el fracaso. En el caso de los colectivistas, esto se traduce en un yo interdependiente, pero diferente de los demás, posiblemente debido a un bajo sentido de libertad e igualdad (Triandis \& Gelfand, 1998). Las personas que se orientan al colectivismo vertical (CV) disfrutan sirviendo a los demás, hacen sacrificios en beneficio de su propio grupo de pertenencia, son modestas y cumplen sus obligaciones como si estuvieran impuestas por normas sociales (Kurman \& Sriram, 2002).

Esta nueva clasificación permitió identificar cuatro tipos de culturas: Individualismo Horizontal (IH) donde las personas se esfuerzan por ser únicas y hacer las cosas a su manera; Individualismo Vertical (IV) la gente quiere hacer las cosas a su manera y se esfuerza por hacerlas mejor que los demás; Colectivismo Horizontal (CH) los objetivos del individuo se confunden o fusionan con los de su grupo de pertenencia y, Colectivismo Vertical (CV) donde la gente se somete a la autoridad de su grupo y está dispuesta a sacrificarse por los intereses grupales (Triandis, 2001). Aunque, esta tipología fue inicialmente propuesta para facilitar comparaciones entre culturas, está demostrando (Triandis \& Suh, 2002) ser un enfoque muy útil para profundizar el conocimiento de las variaciones del colectivismo y el individualismo dentro de una misma cultura, así como analizar las posibles implicaciones psicológicas de la orientación hacia el individualismo o el colectivismo, ya sea vertical u horizontal. Los hallazgos de Oyserman, Coon y Kemmelmeier (2002), por ejemplo, indican que el CH se asocia con el bienestar y la interacción social, en tanto que el IV lo hace con el autoconcepto y los estilos cognitivos. Dayan, Doyle y Markiewicz (2001) señalan que los CH tienen fuertes necesidades de afiliación y se inclinan por la búsqueda de apoyo social como estrategia de coping, en tanto que los IV y los IH son más asertivos, aunque su autoestima se consolida a través del éxito y de los logros obtenidos (Nelson \& Shavitt, 2002). En términos generales, el individualismo, sea vertical, sea horizontal, aparece asociado con desventajas psicológicas y sociales. Scott, Ciarrochi y Deane (2004) han puntualizado que las personas que tienen fuertes valores y creencias individualistas cuentan con menos redes de apoyo social, menores habilidades para manejar las emociones propias y ajenas, menores intenciones de buscar ayuda entre los amigos y los miembros de la familia y más altos niveles de desesperanza e ideas suicidas.

En cuanto a las posibles vinculaciones entre los valores personales y el compromiso laboral, la evidencia empírica muestra un panorama controversial. Algunos autores han argumentado que la cultura puede influir sobre el compromiso del empleado mediante el desarrollo de creencias sobre las actitudes que se esperan de él. En este sentido, Cole, Kalleberg y Lincoln (1993) ponen como ejemplo las empresas japonesas, conocidas mundialmente por sus prácticas de incentivo en la participación de los empleados por medio de programas de mejoramiento continuo 
de la calidad; mientras que Parkes, Bochner y Schneider (2001) explican que el compromiso surge como una retribución a las relaciones más familiares y protectoras entre patrones y empleados que se establecen en las culturas colectivistas. Otros autores (Palich, Hom \& Griffeth, 1995), en cambio, han puesto en duda cualquier tipo de relación entre valores personales y CO. Tales controversias ameritan la ejecución de nuevos estudios que, por un lado, incluyan las tres facetas del CO (ya que hasta la fecha la mayoría ha focalizado sobre la dimensión afectiva del constructo, dejando de lado las facetas normativa y calculativa) y que, por otro lado, se concentren en las diferencias axiológicas intrapaíses, ya que la tendencia más reciente (Shavitt, Zhang, Torelli \& Lalwani, 2006) indica que los valores culturales de los individuos difieren dentro de las culturas nacionales.

Frente a este escenario, el propósito de la presente investigación es contribuir a un mejor conocimiento del rol del individualismo y del colectivismo vertical y horizontal sobre el CO. Más específicamente: a) verificar la capacidad de la reconceptualización axiológica de Triandis (IV-IH-CV-CH) para detectar diferencias en la orientación cultural de las personas dentro de una misma cultura, y b) examinar cómo influye la orientación cultural sobre el tipo y grado de compromiso que vincula al empleado con su organización. Sobre todo porque hasta la fecha, la investigación ha estado dedicada al estudio del individualismo y del colectivismo entre culturas (Dutta-Bergman \& Wells, 2002; Ferreira et al., 2006; Omar et al., 2007) y podría ser enriquecedor emplear la tipología del individualismo y del colectivismo vertical y horizontal dentro de una misma cultura a la luz de su impacto sobre el mal/bienestar y la in/satisfacción de los empleados.

\section{Método}

\section{Participantes y procedimiento}

Se estudió una muestra por disponibilidad (Tabla 1), integrada por 429 empleados argentinos (228 varones y 201 mujeres) de 12 empresas (2 bancos, 1 asegurador de fondos de pensiones, 1 panificadora, 1 fábrica de tornillos, 2 fábricas de maquinarias agrícolas, 1 papelera, 1 fábrica de cerámica, 2 textiles y 1 fábrica de muebles), radicadas en las zonas centro y sur de Argentina. El promedio de edad fue de 35,2 años y la antigüedad laboral promedio fue de 4,6 años. El 82\% de la muestra tenía estudios secundarios completos o universitarios incompletos y el $18 \%$ restante tenía estudios universitarios completos y ocupaba puestos gerenciales o de cierta responsabilidad. Los sujetos fueron contactados en sus respectivos lugares de trabajo y, luego de hacerles conocer los objetivos del estudio, se los invitó a responder un cuadernillo integrado por una hoja de datos personales y por los reactivos que se describen a continuación. Se les garantizó el anonimato y el empleo confidencial de la información suministrada. 
Tabla 1. Características sociodemográficas de la muestra $(\mathrm{N}=429)$

\begin{tabular}{lll}
\hline Variables sociodemográficas & (\%) \\
\hline Sexo & Varones & 53 \\
& Mujeres & 47 \\
\hline Escolaridad & secundario completo & 52 \\
& universitario incompleto & 30 \\
& universitario completo & 18 \\
\hline Cargo & no gerencial & 82 \\
& gerencial & 18 \\
\hline Tamaño de la empresa & hasta 30 empleados & 27 \\
& de 31 a 60 empleados & 45 \\
\hline Edad & de 61 a 100 empleados & 28 \\
\hline Antigüedad & media= 35,20; s=3,65 & \\
\hline & media= 4,60; s=1,20 & \\
\hline
\end{tabular}

\section{Instrumentos}

\section{Variables personales}

A cada empleado se le solicitó información acerca de su edad, género, escolaridad, cargo y antigüedad laboral. La edad y la antigüedad laboral fueron medidas por extenso. Género y cargo fueron operacionalizadas como variables dummy: (1= varón, 2= mujer) y (1= no gerencial y $2=$ gerencial), respectivamente. Se empleó una codificación basada en escalas de tres puntos para la operacionalización del nivel de escolaridad (1= secundario completo, $2=$ universitario incompleto y $3=$ universitario completo) y el tamaño de la empresa (1= hasta 30 empleados; $2=$ de 31 a 60 empleados; $3=$ de 61 a 100 empleados).

\section{Colectivismo e individualismo vertical y horizontal}

La orientación axiológica fue explorada mediante la versión validada por Omar (2005) de la Escala de Individualismo-Colectivismo desarrollada por Singelis, Triandis, Bhawuk y Gelfand (1995). Integrada por 32 ítems con formato Likert de 5 puntos, variando de 1 (totalmente en desacuerdo) a 5 (totalmente de acuerdo), 
la escala evalúa cada una de las cuatro dimensiones del individualismocolectivismo, a través de 8 ítems cada una. La dimensión IH enfatiza la independencia y la autonomía (ej.: "me gusta ser único y diferente a los otros de muchas maneras", "prefiero ser directo y franco cuando discuto con la gente”). La dimensión IV enfatiza el poder y la competencia (ej.: "ganar es todo”, "para mi es importante hacer mi trabajo mejor que los demás”). La dimensión CH se caracteriza por la cooperación y la preservación de la armonía grupal (ej.: "me siento bien cuando colaboro con los demás”, “para mí es importante mantener la armonía dentro de mi grupo”). La dimensión CV privilegia las metas grupales sobre las individuales y el respeto por superiores y autoridades (ej.: "haría lo que agrade a mi familia, aún cuando a mí no me guste esa actividad”, "a los niños se les debe enseñar el deber antes que el placer”). El índice de consistencia interna (alpha de Cronbach) de la escala total fue de á=0,84 y las dimensiones presentaron índices superiores a 0,75 .

\section{Compromiso Organizacional}

El CO fue explorado a través de la escala tridimensional desarrollada por Allen y Meyer (1996), validada para su empleo con muestras argentinas por Omar (2005). Integrada por 18 ítems con formato Likert de 5 puntos, variando de 1 (totalmente en desacuerdo) a 5 (totalmente de acuerdo), la escala evalúa cada uno de los tres tipos de CO, a través de 6 ítems cada uno. El compromiso afectivo (ej.: "estaría feliz si pasara el resto de mi carrera en la empresa donde trabajo", "siento como si los problemas de la empresa fueran míos”); el compromiso normativo (ej.: "me sentiría culpable si abandono mi empresa”, "esta organización merece mi lealtad”), y el compromiso calculativo (ej. : "siento que tengo pocas opciones de trabajo como para dejar mi organización”, "si no hubiera dado tanto de mí a la empresa, podría considerar cambiar de trabajo”). Los índices de consistencia interna (alpha de Cronbach) de la versión empleada en el presente estudio fueron de 0,82 para el compromiso afectivo; 0,73 para el normativo y 0,76 para el calculativo.

\section{Resultados}

En primer lugar, y considerando la muestra inicial de 429 empleados, se calcularon las medianas, medias y desvíos típicos correspondientes a cada una de las orientaciones axiológicas exploradas mediante la escala de Singelis et al. (1995). En la Tabla 2 se presentan los respectivos índices descriptivos. 
Tabla 2. Índices descriptivos correspondientes a cada una de las orientaciones axiológicas $(\mathrm{N}=429)$

\begin{tabular}{lccc}
\hline Orientaciones axiológicas & Mediana & Media & Desvío \\
\hline Individualismo Horizontal (IH) & 3.10 & 3.19 & 0.90 \\
\hline Individualismo Vertical (IV) & 2.05 & 2.11 & 0.81 \\
\hline Colectivismo Horizontal (CH) & 3.65 & 3.69 & 0.86 \\
\hline Colectivismo Vertical (CV) & 3.30 & 3.43 & 1.01 \\
\hline
\end{tabular}

A continuación, y con el propósito de explorar las relaciones entre la orientación cultural dominante de cada empleado y el CO con su empresa, se procedió a dicotomizar cada variable axiológica en función del valor de la mediana. Este procedimiento permitió conformar un total de 8 subgrupos. A partir de estos 8 subgrupos, se seleccionaron los cuatro más representativos, considerándose como representativo el grupo que exhibía altos puntajes en sólo una de las 4 dimensiones axiológicas y bajo en las restantes tres dimensiones axiológicas. Los cuatro subgrupos representativos de IH, IV, CH y CV, quedaron integrados por 36, 29, 45 y 52 sujetos, respectivamente, totalizando 162 sujetos. Por lo que a posteriori, se analizaron las relaciones entre la orientación cultural y los tipos de CO solamente en estos cuatro subgrupos. Dentro de cada subgrupo axiológico se calculó la media de cada tipo de CO (Tabla 3), información que fue empleada para el cálculo de análisis de la varianza simple (ANOVA).

Tabla 3. Medias de los diferentes tipos de compromiso organizacional (CO) correspondientes a cada uno de los cuatro subgrupos axiológicos $(\mathrm{N}=162)$.

\begin{tabular}{llllll}
\hline & IH & IV & CH & CV & TOTAL \\
\hline CO afectivo & 2.96 & 3.02 & 4.25 & 3.60 & 3,46 \\
\hline CO normativo & 3.85 & 3.16 & 1.92 & 2.53 & 2,87 \\
\hline CO calculativo & 3,79 & 4.07 & 3,21 & 3,16 & 3,56 \\
\hline
\end{tabular}

Los análisis de la varianza (ANOVA), ejecutados para comparar las diferencias de medias de tres o más grupos simultáneamente, revelaron que tanto el compromiso afectivo $\left(\mathrm{F}_{(3,159)}=5,72 ; \mathrm{p}=0,001\right)$, como el compromiso normativo $\left(\mathrm{F}_{(3,159)}=3,87 ; \mathrm{p}=0,01\right)$ fue significativamente diferente entre los cuatro grupos. Comparaciones a posteriori, mediante el test de Tukey mostraron que los sujetos del grupo CH tenían más compromiso afectivo 
(media $=4.25)$ que los del grupo de CV (media $=3.60 ; \mathrm{p}<0,01)$ y que los grupos de individualistas $(\mathrm{p}=0,02)$. En tanto que los sujetos del grupo IV reconocían más compromiso calculativo (media $=4.07$ ) que los restantes subgrupos axiológicos. En términos generales, al ordenar los subgrupos axiológicos en función del monto de compromiso afectivo $(\mathrm{CH}>\mathrm{CV}>\mathrm{IV}>\mathrm{IH}$ ), se observó que este tipo de compromiso es mayor entre los colectivistas que entre los individualistas. En cambio, tanto el compromiso normativo ( $\mathrm{IH}>\mathrm{IV}>\mathrm{CV}>\mathrm{CH}$ ), como el calculativo $(\mathrm{IV}>\mathrm{IH}>\mathrm{CH}>\mathrm{CV}$ ) mostraron ser más fuertes entre los individualistas que entre los colectivistas.

Finalmente, a efectos de verificar la incidencia de los factores socioculturales sobre el compromiso entre los diferentes subgrupos axiológicos, se procedió a calcular los coeficientes de correlación entre las variables exploradas. La Tabla 4 muestra la matriz de correlaciones entre la totalidad de las variables consideradas.

Tabla 4. Matriz de correlaciones entre la totalidad de las variables estudiadas $(\mathrm{N}=162)$

\begin{tabular}{|c|c|c|c|c|c|c|c|c|c|c|c|c|}
\hline & (1) & (2) & (3) & (4) & (5) & (6) & (7) & (8) & (9) & (10) & (11) & (12) \\
\hline (1) $H$ & 一 & .019 & .002 & .010 & .114 & $.367^{* *}$ & $.297 * \star$ & .035 & .110 & $.251^{* *}$ & .147 & .165 \\
\hline (2) IV & & 一 & .101 & .097 & .124 & $.286 * *$ & $.344^{\star *}$ & .056 & .059 & $.307^{\star \star}$ & .116 & .157 \\
\hline (3) $\mathrm{CH}$ & & & - & .116 & $.320 * *$ & $.197^{\star}$ & .121 & .101 & $.184^{\star}$ & $-.185^{\star}$ & .098 & $-.340 * *$ \\
\hline (4) CV & & & & - & $.278^{* *}$ & $.181 *$ & .109 & .018 & .172 & -.133 & .134 & $.276^{\star *}$ \\
\hline (5)C afectivo & & & & & - & .015 & .077 & $.527^{\star *}$ & $.471^{* *}$ & $-.409 * *$ & $-.216^{\star \star}$ & $-.521^{* *}$ \\
\hline (6) C normativo & & & & & & - & .104 & $.211^{* *}$ & $.356^{* *}$ & $.287^{\star \star}$ & $.316^{* \star}$ & $.321 * *$ \\
\hline (7)C calculativo & & & & & & & - & .115 & .098 & .144 & .112 & .176 \\
\hline (8) Antigüedad & & & & & & & & - & .165 & $.355^{* *}$ & .098 & $.223^{* *}$ \\
\hline (9) Género & & & & & & & & & 一 & $-.182^{\star}$ & .178 & .118 \\
\hline (10) Cargo & & & & & & & & & & 一 & $.618^{\star *}$ & .127 \\
\hline (11)Escolaridad & & & & & & & & & & & - & .174 \\
\hline
\end{tabular}

(12) Tamaño E.

$$
{ }^{*} \mathrm{p}<0,01 ; * * \mathrm{P}<0,001
$$

Como se desprende de la tabla precedente, los coeficientes de correlación obtenidos confirman la mayor tendencia al compromiso afectivo por parte de los colectivistas (horizontales y verticales), así como la prevalencia del compromiso normativo y/o 
calculativo entre los individualistas (horizontales y verticales). En lo que hace a las relaciones entre las características sociodemográficas de los empleados y el tipo de CO que los vincula con su empresa, se observa que el compromiso afectivo se vincula positivamente con la antigüedad y el género de los empleados, aunque negativamente con el cargo, el nivel de escolaridad y el tamaño de la empresa. A su vez, mientras que el compromiso normativo se asocia positiva y significativamente con la totalidad de las variables sociodemográficas consideradas, el compromiso calculativo no presenta asociaciones significativas con tales variables.

\section{Discusión}

Los valores han sido definidos como principios que guían la vida (Schwartz, 1992) y orientan la conducta de las personas, incluyendo las asociadas con el trabajo (Schwartz, 1999). De allí que muchos estudios actuales están focalizados sobre la congruencia entre los valores de los individuos y los de las organizaciones donde trabajan, en sintonía con las observaciones de Dose (1997) quien señala que como 'gran parte de nuestro tiempo transcurre en entornos laborales’ (p. 236), los valores asociados al trabajo son particularmente importantes y relevantes. Y si bien la orientación cultural ha sido abordada predominantemente en estudios transculturales, la tendencia actual se orienta al análisis de los valores individuales dentro de una misma cultura. El presente estudio se inscribe en esta tendencia, y con tal propósito se ha adoptado el esquema clasificatorio propuesto por Triandis, basado en los cuatro tipos axiológicos (IH, IV, CH y CV), como herramienta idónea para examinar el rol de la orientación cultural individual sobre el tipo y grado de compromiso que vincula al empleado con su organización.

A partir de la clasificación de los sujetos en cuatro grupos de acuerdo a su predisposición cultural, los resultados obtenidos confirman y clarifican las vinculaciones entre la orientación axiológica individual y el CO. Aunque clasificados como una cultura colectivista por investigaciones previas (Hofstede, 1980, 1997; Schwartz, 1994), los miembros de la cultura argentina exhiben diferencias en cuanto a sus orientaciones culturales lo que, a su vez, explicaría sus diferentes tipos de compromiso con la organización donde se desempeñan. Al respecto, pareciera que los empleados con mayor orientación al colectivismo, sea horizontal, sea vertical, desarrollan mayor nivel de compromiso afectivo. En tanto que los empleados más orientados al individualismo, se vinculan con sus organizaciones a través del compromiso normativo y/o calculativo.

En lo que hace a las variables sociodemográficas, y en coincidencia con lo informado por Van Breukelen, Van der Vlist y Steensma (2004), son las mujeres y los empleados con mayor antigüedad quienes exhiben mayores niveles de compromiso afectivo y normativo. Los empleados menos escolarizados y los que ocupan cargos de menor jerarquía se sienten unidos a sus empresas a través de lazos afectivos, a diferencia de los que han completado sus estudios superiores y de los que ocupan cargos gerenciales, quienes reconocen que su permanencia en la organización obedece a sentimientos de deber, lealtad y reconocimiento por lo que la empresa ha hecho por ellos (compromiso 
normativo). Por su parte, los empleados de empresas pequeñas (hasta 30 empleados) y medianas (entre 31 y 60 empleados) demuestran mayor compromiso afectivo que los empleados de empresas grandes (más de 61 empleados), quienes a su vez, se sienten unidos a sus organizaciones a través del compromiso normativo (sentimientos de deber y obligación de permanecer en la empresa). Estas vinculaciones entre CO y tamaño de la empresa están en sintonía con los hallazgos comunicados recientemente por Latting et al. (2004), quienes observan que los grupos pequeños incrementan las interacciones sociales y contribuyen al desarrollo y fortalecimiento del compromiso afectivo entre sus miembros. Asimismo, el tamaño de la empresa presenta asociaciones significativas con la orientación axiológica del empleado, desde el momento que los colectivistas (horizontales o verticales) parecen preferir las empresas pequeñas o medianas. Posiblemente, porque encuentran que en las empresas pequeñas se fortalecen sus necesidades de trabajar en grupo, de contar con suficiente apoyo emocional por parte de los compañeros, de experimentar mayores sentimientos de satisfacción y bienestar personal. En este sentido, resultan particularmente ilustrativas las observaciones de Finegan (2000), cuando señala que las empresas desarrollan sus propias culturas y tienden a atraer gente con similares características, incluyendo sus valores.

El compromiso calculativo, por su parte, pareciera operar de modo diferente al normativo y al afectivo, desde el momento que no aparece vinculado con ninguna de las variables sociodemográficas exploradas. Frente a este comportamiento diferencial, la pregunta si el compromiso calculativo constituye una verdadera faceta del CO (Meyer et al., 2002) vuelve a ocupar el centro de la escena. Por lo que se imponen nuevas investigaciones con el objetivo de verificar su pertenencia (o no) a la red nomológica del constructo.

Para evaluar los hallazgos y las contribuciones del presente estudio se deben tomar en cuenta las limitaciones de su diseño. Al respecto, no hay que perder de vista que todas las variables fueron medidas al mismo tiempo y con la misma muestra, lo que puede haber ocasionado algunos sesgos de medición. A su vez, como los datos fueron recolectados en un mismo país, habría que tener cautela al momento de intentar generalizar los resultados obtenidos, sobre todo los vinculados con los colectivistas. Desde el trabajo pionero de Hofstede (1980), Argentina se está ubicando entre los países colectivistas (Schwartz, 1994). Sin embargo, y tal como lo ha señalado Kagitcibasi (1997), a medida que crece la urbanización de los países en vías de desarrollo decrece la interdependencia intergeneracional. Circunstancia que contribuye a disminuir el colectivismo y aumentar el individualismo. Por lo tanto es importante considerar si esta particular circunstancia tiene alguna implicancia sobre los resultados del presente estudio. Finalmente, si bien esta investigación provee información preliminar sobre las relaciones entre orientación cultural y CO se necesitarían nuevas verificaciones para entender y explicar mejor el rol de cada una de las orientaciones axiológicas en la determinación de estos particulares estados psicológicos dentro de una misma cultura. Sobre el particular, el empleo de enfoques cualitativos basados en observaciones empíricas en el lugar de trabajo podría constituirse en contribuciones importantes. 


\section{Referencias}

Alavi, S.B. \& McCormick, J. (2004). Theoretical and measurement issues for studies of collective orientation in team contexts, Small Group Research, 35, 2, 111-127.

Allen, N. J., \& Meyer, J. P. (1996). Affective, continuance and normative commitment to the organization: An examination of construct validity. Journal of Vocational Behavior, 49, 252-276.

Clugston, M., Howell, J.P., \& Dorfman, P.W. (2000). Does cultural socialization predict multiple bases and foci of commitment? Journal of Management, 26, 5-30.

Cole, R.E., Kalleberg, A.L., \& Lincoln, J.R. (1993). Assessing commitment in the United States and Japan: A comment on Besser. American Sociological Review, 58, 882-885.

Dayan, J., Doyle, AB., \& Markiewicz, D. (2001). Social support networks and selfesteem of idiocentric and allocentric children and adolescents. Journal of Social \& Personal Relationships, 18, 767-784.

Dose, J.J. (1997). Work values: An integrative framework and illustrative application to organizational socialization. Journal of Occupational and Organizational Psychology, 70, 219-240.

Dutta-Bergman, M. J., \& Wells, W. D. (2002). The values and lifestyles of idiocentrics and allocentrics in an individualist culture: A descriptive approach. Journal of Consumer Psychology, 12, 3, 231-242.

Ferreira, C., Assmar, E.; Omar, A.; Uribe, H., Terrones, A, \& Galaz, M. (2006). Individualismo e colectivismo, percepçòes de justiça e comprometimento em organizaçóes latino-americanas. Revista Interamericana de Psicología, 40, 2, 53-62.

Finegan, J.E. (2000). The impact of person and organizational values on organizational commitment. Journal of Occupational and Organizational Psychology, 73, 2, 149-169.

Greenberg, J., \& Baron, R. A. (1997). Behavior in organizations. New Jersey: Prentice-Hall

Hattrup, K.; Mueller, K., \& Joems, I. (2007). The effects of nations and organizations on work value importance: a cross-cultural investigation. Applied Psychology: An International Review, 56, 3, 479-499.

Hofstede, G. (1980). Culture's consequences. New York: Sage.

Hofstede, G. (1997). Cultures and organizations: Software of the mind. New York: McGraw Hill.

Kagitcibasi, C. (1997). Individualism and collectivism. In J.W. Berry, M.H. Segall, \& C. Kagitcibasi (Eds.). Handbook of Cross-Cultural Psychology (pp. 2-49). Boston: Allyn \& Bacon. 
Kurman, J., \& Sriram, N. (2002). Interrelationships among vertical and horizontal collectivism, modesty, and self-enhancement. Journal of Cross-Cultural Psychology, 33, 1, 71-86.

Latting, J.K., Beck, M.H., Slack, K.J., Tetrick, L.E., Jones, A.P., Etchegaray, J.M., \& Da Silva, N. (2004). Promoting service quality and client adherence to the service plan: The role of top management's support for innovation and learning. Administration in Social Work, 28, 2, 29-48.

Mathieu, J.E., \& Zajac, D.M. (1990). A review and meta-analysis of the antecedents, and consequences of organizational commitment. Psychological Bulletin, 108, 171-194.

Meyer, J.P., \& Allen, N.J. (1991). A three component conceptualization of organizational commitment. Human Resource Management Review, 1, 61-89.

Meyer, J.P., Allen, N.J. \& Smith, S. (1993). Commitment to organizations and occupations: Extension and test of a three-component conceptualization. Journal of Applied Psychology, 78, 538-551.

Meyer, J.P., Stanley, D.J., Herscovitch, L., \& Topolnytsky, L. (2002). Affective, continuance and normative commitment to the organization: A meta-analysis of antecedents, correlates and consequences. Journal of Vocational Behavior, 61, $20-52$.

Nelson, M.R., \& Shavitt, S. (2002). Horizontal and vertical individualism and achievement values. A multimethod examination of Denmark and United States. Journal of Cross-Cultural Psychology, 33, 439-458.

Omar, A. (2005). La cultura organizacional de las empresas argentinas. Rosario: Conicet.

Omar, A.; Uribe, H., Ferreira, C., Assmar, E., Terrones, A., \& Galaz, M. (2007). Colectivismo, justicia y ciudadanía organizacional en empresas argentinas, mexicanas y brasileras. Revista Mexicana de Psicología, 24, 1, 101-116.

Oyserman, D., Coon, H., \& Kemmelmeier, M. (2002). Rethinking individualism and collectivism: Evaluation of theoretical assumptions and meta-analyses. Psychological Bulletin, 128, 3-73.

Palich, L.E., Hom, P.W., \& Griffeth, R.W. (1995). Managing in the international context: Testing the cultural generality of sources of commitment to multinational enterprises. Journal of Management, 21, 671-690.

Parkes, L.P., Bochner, S., \& Schneider, S.K. (2001). Person-organization fit across cultures: An empirical investigation of individualism and colectivism. Applied Psychology: An International Review, 50, 81-108.

Ramamoorthy, N., \& Flood, P. (2002). Employee attitudes and behavioral intentions: A test of the main and moderating effects of individualism-collectivism orientations. Human Relations, 55, 1071-1096. 
Schwartz, S.H. (1992). Universals in the content and structure of values: Theoretical advances and empirical tests in 20 countries. Advances in Experimental Social Psychology, 25, 1-65.

Schwartz, S.H. (1994). Beyond individualism-collectivism: New cultural dimensions of values. In U. Kim, H. Triandis, C. Kagitcibasi, S.C. Choi, \& G. Yoon (Eds.). Individualism and collectivism: Theory, method and applications (pp. 85-119). Thousand Oaks, CA: Sage.

Schwartz, S.H. (1999). A theory of cultural values and some implications for work. Applied Psychology: An International Review, 48, 1, 23-47.

Scott, G., Ciarrochi, J. \& Deane, F. (2004). Disadvantages of being an individualist in an individualistic culture: Idiocentrism, emotional competence, stress, and mental health. Australian Psychologist, 39, 2, 143-153.

Shavitt, S., Zhang, J., Torelli, J., \& Lalwani, A.K. (2006). Reflections on the meaning and structure of the horizontal/vertical distinction. Journal of Consumer Psychology, $16,4,3547-362$.

Singelis, T.M., Triandis, H.C., Bhawuk, D., \& Gelfand, M.J. (1995). Horizontal and vertical dimensions of individualism and collectivism: A theoretical and measurement refinement. Cross-Cultural Research, 29, 240-275.

Triandis, H.C. (1995). Individualism and collectivism. Boulder, CO: Westview.

Triandis, H.C. (2001). Individualism-collectivism and personality. Journal of Personality, 69, 907-924.

Triandis, H.C., \& Gelfand, M.J. (1998). Converging measurement of horizontal and vertical individualism and collectivism. Journal of Personality and Social Psychology, $74,1,118-128$.

Triandis, H.C., \& Suh, E.M. (2002). Cultural influences on personality. Annual Review of Psychology, 53, 133-160.

Triandis, H.C., Chen, X.P., \& Chan, D.K. (1998). Scenarios for the measurement of collectivism and individualism. Journal of Cross-Cultural Psychology, 29, 2, 275-289.

Trice, H.M. \& Beyer, J.M. (1993). The culture of work organizations. Englewood Cliffs, NJ: Prentice Hall.

Van Breukelen, W., Van Der Vlist, R., \& Steensma, H. (2004). Voluntary employee turnover: combining variables from the 'traditional' turnover literature with the theory of planned behavior. Journal of Organizational Behavior, 25, 7, 893-914.

Virtanen, T. (2000). Commitment and the study of organizational culture and climate. In N.M. Ashkanasy, C.P.M. Wilderom \& M.F. Peterson (Eds.), Handbook of organizational culture and climate (pp. 339-354). Thousand. 
Psicodebate 9. Psicología, Cultura y Sociedad 